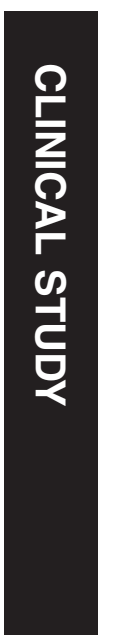

\section{Risk of intraocular hemorrhage with new oral anticoagulants}

\author{
Abstract \\ Purpose To assess the risk of intraocular \\ hemorrhage with warfarin and new oral \\ anticoagulants (NOACs). \\ Methods We ascertained all reported cases \\ of intraocular hemorrhage (vitreous, \\ choroidal, or retinal) with warfarin and \\ NOACs (including dabigatran, rivaroxaban, \\ apixaban) from the World Health \\ Organizations's Vigibase database from \\ 1968-2015. We used a disproportionality \\ analysis to compute reported odds ratios \\ (RORs) and corresponding $95 \%$ confidence by \\ comparing the number of events with the \\ study outcomes and study drugs compared \\ with all other drugs reported to Vigibase. \\ A harmful signal was deemed for a lower \\ limit of the 95\% confidence interval above 1 . \\ Results We identified 80 cases of intraocular \\ hemorrhage (vitreous, choroidal, or retinal) \\ with warfarin in the World Health \\ Organizations's Vigibase database from \\ 1968-2015. A total of 156 cases of intraocular \\ hemorrhage with NOACs (82 with \\ rivaroxaban, 65 with dabigatran, 9 with \\ apixaban). Warfarin had the highest signal of \\ association with choroidal hemorrhage \\ $(\mathrm{ROR}=65.40$ (33.86-126.30)). Rivaroxaban \\ had the highest signal of association with \\ both retinal and vitreous hemorrhage \\ $(\mathrm{ROR}=7.41$ (5.73-9.59) and $\mathrm{ROR}=11.14$ \\ (7.37-16.86), respectively). Dabigatran was \\ also significantly associated with retinal and \\ vitreous hemorrhage $(\mathrm{ROR}=3.78(2.82-5.08)$ \\ and $\mathrm{ROR}=5.83$ (3.66-9.30), respectively). The \\ number of reports of retinal and vitreous \\ hemorrhage were also significantly higher \\ with apixaban, but the number of cases may \\ be too little to make a meaningful evaluation. \\ Conclusion A signal for risk of intraocular \\ hemorrhage was detected for warfarin, \\ dabigatran, and rivaroxaban. Large \\ epidemiologic studies are needed to further \\ confirm these findings. \\ Eye (2017) 31, 628-631; doi:10.1038/eye.2016.265; \\ published online 23 December 2016
} University of British Columbia, Vancouver, BC, Canada

${ }^{2}$ Department of Pharmacology and Therapeutics, University of British Columbia,

Vancouver,

BC, Canada

${ }^{3}$ Department of Ophthalmology and Visual Sciences, University of British Columbia, Vancouver, BC, Canada

Correspondence: M Etminan, Assistant Department of Ophthalmology and Visual Sciences, The University of British Columbia, The Eye Care Center, Room 323-2550 Willow Street, Vancouver BC, V5Z 3N9 Canada

Tel: +604875 4725;

Fax: +604875 4663 .

E-mail: etminanm@

mail.ubc.ca

Received: 13 June 2016 Accepted in revised form: 6 October 2016

Published online: 23 December 2016
G Talany ${ }^{1}, \mathrm{M} \mathrm{Guo}^{1,2}$ and M Etminan ${ }^{1,2,3}$

\section{Introduction}

New oral anticoagulants (NOACs) such as dabigatran, rivaroxaban and apixaban have become popular medications among clinicians. In 2014, rivaroxaban accounted for $\sim \$ 1.5$ billion in sales, which represented a $76 \%$ increase compared with 2013. ${ }^{1}$ The main advantage of NOACs compared with warfarin is the lack of coagulation monitoring or dosage adjustments required due to more predictable pharmacokinetic properties. ${ }^{2-4}$ Recent studies have examined the risk of bleeding with these drugs, however, information on the risk of ocular bleeding with NOACs is scant.

Anticoagulants help reduce the risk of deep vein thrombosis, pulmonary embolism, myocardial infarction, and strokes. ${ }^{4}$ In relation to ophthalmic indications, anticoagulants have been used in the treatment of coagulation abnormalities (such as protein $\mathrm{C}$ or $\mathrm{S}$ or antithrombin III deficiencies), and in patients with ischemic optic neuropathies. Despite this reduced risk of serious thromboembolic phenomena, anticoagulants increase the risk of hemorrhage. NOACs have also been associated with an increased risk of hemorrhage due to their mechanisms of binding and neutralizing clotting factors or components of the coagulation cascade. ${ }^{5}$ Although this reduces the ability for blood to clot and leads to the drug's therapeutic potential, NOACs might also lead to a higher propensity for hemorrhaging without an available antidote. ${ }^{4,6}$

Operating on an anticoagulated patient has been associated with bloody tears, hyphemas, vitreal, subconjunctival, subretinal, and choroidal hemorrhages. ${ }^{7-12}$ Although most reports of intraocular hemorrhage relate to warfarin, the same effects have been reported less frequently for NOACs. ${ }^{13-15}$ One welldescribed case report has linked rivaroxaban to spontaneous vitreous hemorrhage. ${ }^{16}$ Despite epidemiologic studies demonstrating a link between oral anticoagulants and intraocular 
hemorrhage, to our knowledge, no study to date has examined and compared the risk of intraocular hemorrhage with warfarin and NOACs.

\section{Materials and methods}

We ascertained all reported cases of intraocular hemorrhage (vitreous, choroidal, or retinal) with warfarin and NOACs (including dabigatran, rivaroxaban, and apixaban) from the World Health Organizations's Vigibase database from 1968-2015. Besides the pharmaceutical product name and its associated adverse event, the reports do not always include other information such as concomitant disease. 11582092 records of spontaneous adverse events were captured in the database at the time of study, which were all included in the disproportionality analysis.

We used a disproportionality analysis to compute reported odds ratios (RORs) and corresponding 95\% confidence by comparing the number of events with the study outcomes and study drugs compared with all other drugs reported to Vigibase. First, the data mining is performed through drug monitoring databases like the WHO's adverse events reporting database to acquire all relevant reports of the adverse event and the drug of interest. Then, the number of reports of an event of interest associated with the drug of interest is compared with the number of reports of all other adverse events associated with this drug as well as the number of reports of this event associated with all other drugs listed in the database. We only included cases that reported taking one of the three NOAC medications; cases where two drugs were taken concomitantly were excluded. This approach examines whether the number of reports of the event is statistically higher with the drug in question ws with all other drugs in the database. ${ }^{17} \mathrm{~A}$ harmful signal was deemed for a lower limit of the $95 \%$ confidence interval above $1 .{ }^{18}$ Since this is a hypothesis-generating observational study, statistical power is ascertained from the width of the confidence intervals. If results are statistically significant (that is, the lower bound exceeds $1)$, adequate statistical power is reached.

\section{Results}

We identified 80 cases of intraocular hemorrhage (vitreous, choroidal, or retinal) with warfarin in the World Health Organizations's Vigibase database from 1968-2015 (Table 1). A total of 156 cases of intraocular hemorrhage with NOACs (82 cases with rivaroxaban, 65 with dabigatran, and 9 with apixaban). Warfarin had the highest signal of association with choroidal hemorrhage $(\mathrm{ROR}=65.40$ (33.86-126.30)). Rivaroxaban had the highest signal of association with both retinal and
Table 1 Reporting odds ratios calculated for cases of choroidal, retinal, and vitreous hemorrhage associated with common anticoagulants

\begin{tabular}{lccc}
\hline Drug name/condition & $\begin{array}{c}\text { Number of } \\
\text { events }\end{array}$ & $\begin{array}{c}\text { Reporting } \\
\text { odds ratio }\end{array}$ & $\begin{array}{c}\text { 95\% confidence } \\
\text { interval }\end{array}$ \\
\hline $\begin{array}{l}\text { Choroidal Hemorrhage } \\
\quad \text { Warfarin }\end{array}$ & 12 & 65.40 & $(33.86-126.30)$ \\
$\quad$ Dabigatran & 2 & 13.29 & $(3.22-54.82)$ \\
& & & \\
Retinal hemorrhage & & & \\
$\quad$ Warfarin & 50 & 2.67 & $(1.39-2.30)$ \\
$\quad$ Dabigatran & 45 & 3.78 & $(2.82-5.08)$ \\
Apixaban & 7 & 3.25 & $(1.55-6.83)$ \\
$\quad$ Rivaroxaban & 59 & 7.41 & $(5.73-9.59)$ \\
& & & \\
Vitreous hemorrhage & & & \\
$\quad$ Warfarin & 18 & 3.69 & $(2.32-5.89)$ \\
$\quad \begin{array}{l}\text { Dabigatran } \\
\text { Apixaban }\end{array}$ & 18 & 5.83 & $(3.66-9.30)$ \\
Rivaroxaban & 2 & 3.55 & $(0.89-14.24)$ \\
& 23 & 11.14 & $(7.37-16.86)$ \\
\hline
\end{tabular}

vitreous hemorrhage $(\mathrm{ROR}=7.41$ (5.73-9.59) and $\mathrm{ROR}=11.14$ (7.37-16.86), respectively). Dabigatran was also significantly associated with retinal and vitreous hemorrhage $(\mathrm{ROR}=3.78(2.82-5.08)$ and $\mathrm{ROR}=5.83$ (3.66-9.30), respectively). See Table 1 for a summary of statistical outputs. The number of reports of retinal and vitreous hemorrhage were also higher with apixaban, but the number of cases may be too little to make a meaningful evaluation.

\section{Discussion}

The results of our study demonstrated a larger proportion of intraocular hemorrhage linked to warfarin and NOACs compared with other drugs in the WHO database. Warfarin had the highest signal of association with choroidal hemorrhage (Table 1). This is likely due to warfarin's longer history of use, hence a heavier predisposition to report hemorrhage incidents with the drug, resulting in a higher number of reported incidents involving choroidal hemorrhage. Rivaroxaban had the highest signal of association with both retinal and vitreous hemorrhage despite being FDA-approved, a year later than dabigatran. This suggests a higher risk of retinal and vitreous hemorrhage in rivaroxaban users compared with dabigatran. Apixaban was least associated with either condition, likely due to a smaller number of reported cases, as apixaban is the latest of the drug class to be approved by the FDA (as of 2012). ${ }^{19}$

There is strong biological evidence as to why Warfarin and NOACs may result in intraocular hemorrhage.

Warfarin inhibits vitamin $\mathrm{K}$ utilization, which reduces the activation of prothrombin complex proteins and the activity of clotting factors, effectively slowing thrombin generation 
and clot formation..$^{2-4}$ NOACs such as rivaroxaban and apixaban are specific inhibitors of factor $\mathrm{Xa}$, and dabigatran is a direct thrombin inhibitor. ${ }^{3-5}$ Reducing the risk of forming a clot increases hemorrhagic diathesis, ${ }^{3-5}$ which increases the risk of intraocular hemorrhage.

Intraocular hemorrhage is a serious adverse event for patients taking anticoagulants. Substantial intraocular hemorrhages can cause severe visual acuity impairment, and in some cases, surgery is needed for complete resolution. ${ }^{20,21}$ Unfortunately, there are no substantial recommendations or guidelines regarding the modification of warfarin and NOACs prior to any type of ocular surgery. The decision to withhold, modify, or continue anticoagulation should be individualized. Consideration of the patient's medical history, specific surgical procedure required, and consultation with the physician responsible for monitoring the patient's anticoagulation is prudent. Finally, patients must be fully informed of the risks involved with either maintenance or discontinuation of anticoagulation therapy.

Use of a disproportionality analysis demonstrated associations with a rare adverse event, which prior randomized trials were unable to quantify due to limited statistical power (small sample size and short follow-up). This technique is frequently used by the FDA and other post marketing surveillance programs to detect adverse drug events. ${ }^{22}$ However, as with all the data mining studies, this study is subject to limitations. Due to reliance on voluntary reporting from patients, healthcare providers and pharmaceutical companies, ${ }^{22}$ and a lack of comprehensive patient data, results from the spontaneous data cannot provide evidence of causation and are meant to be hypothesis-generating.

Further, this approach does not control for confounding by age, indication for drug use, or other factors that may correlate with drug usage. The reports also do not include pertinent visits or follow-ups, so we have no way of knowing the present status of the reported cases nor how long patients were exposed to the drug before developing intraocular bleeds. Under-reporting - as a result of the missing data and reluctance to file voluntary reportswould underestimate the true risk of these rare adverse events, as may be in the case of apixaban (Table 1). This makes the search for adverse events like NOAC-

associated intraocular hemorrhage difficult since there are relatively few reports.

The main limitations in comparing between warfarin and NOACs are (1) the length of time for which warfarin has been used as an anti-coagulant, (2) differences in pharmacokinetics, and (3) potentially different comorbid conditions in use of NOACs and warfarin, which may affect intraocular bleeding. Warfarin likely presents a higher number of adverse event reports than for NOACs due to the reporting bias mentioned above, which would increase its apparent risk of certain adverse events. Although in the same class of drugs, the mechanism by which NOACs are distributed and eliminated from the body are different from warfarin. Hence, preventing a direct comparison in dosage and the resulting risk of adverse events. Further research is needed to study the connection between the pharmacokinetics and risk of intraocular hemorrhage in NOACs.

\section{Conclusion}

The results of our study suggest a signal of increased intraocular hemorrhage risk with the use of warfarin, dabigatran, and rivaroxaban. The anticoagulation mechanisms of these drugs lends to an increased risk of hemorrhage, more specifically in ocular regions. Other NOACs are newer, hence have fewer reports to draw significant signals from. In light of the increasing use of NOACs for a number of diseases worldwide, further investigation into this question should be done using large well-designed epidemiologic studies linked to a comprehensive ophthalmologic registry of adverse events. More detailed patient information such as age, drug history, and disease characteristics in each case would be valuable for finding potential confounders and to further explore the mechanisms behind this adverse association.

\section{Summary}

\section{What was known before}

- New oral anticoagulants such as dabigatran, rivaroxaban, and apixaban have become popular medications among clinicians.

- Operating on an anticoagulated patient has been associated with bloody tears, hyphemas, vitreal, subconjunctival, subretinal, and choroidal hemorrhages.

- No study to date has examined and compared the risk of intraocular hemorrhage with warfarin and NOACs.

What this study adds

- The results of our study suggest an increased risk of intraocular hemorrhage with the use of warfarin, dabigatran, apixaban and rivaroxaban.

\section{Conflict of interest}

The authors declare no conflict of interest.

\section{Acknowledgements}

World Health Organization (WHO) data was received from the WHO Collaborating Centre for International Drug Monitoring in Uppsala, Sweden. The information provided does not represent the opinions of either organization. 


\section{References}

1 Johnson \& Johnson. 2014 Johnson \& Johnson Annual Report. Available at: http:/ / files.shareholder.com/ downloads/JNJ/194526085x0x815170/816798CD-60D94653-BB5A-50A66FD5B9E7/JNJ_2014_Annual_Report_ bookmarked_pdf (accessed on 25 February 2016).

2 Lauffenburger J, Farley J, Gehi A, Rhoney D, Brookhart M, Fang G. Factors driving anticoagulant selection in patients with atrial fibrillation in the united states. Am J Cardiol 2015; 115(8): 1095-1101.

3 Patel MR, Mahaffey KW, Garg J, Pan G, Singer DE, Hacke WROCKET AF Investigators. Rivaroxaban versus warfarin in nonvalvular atrial fibrillation. N Engl J Med 2011; 365(10): 883-891.

4 Wadhera RK, Russell CE, Piazza G. Warfarin versus novel oral anticoagulants: how to choose? Circulation 2014; 130(22): e191-e193.

5 Madan S, Shah S, Partovi S, Parikh SA. Use of novel oral anticoagulant agents in atrial fibrillation: current evidence and future perspective. Cardiovasc Diagn Ther 2014; 4(4): 314-323.

6 Caldeira D, Canastro M, Barra M, Ferreira A, Costa J, Pinto FJ et al. Risk of substantial intraocular bleeding with novel oral anticoagulants: Systematic review and metaanalysis. JAMA Ophthalmol 2015; 133(7): 834-839.

7 Weir CR, Nolan DJ, Holding D, Hammer H. Intraocular haemorrhage associated with anticoagulant therapy. Acta Ophthalmol Scand 2000; 78(4): 492-493.

8 Bloome MA, Ruiz RS. Massive spontaneous subretinal hemorrhage. Am J Ophthalmol 1978; 86(5): 630.

9 Brown GC, Tasman WS, Shields JA. Massive subretinal hemorrhage and anticoagulant therapy. Can J Ophtalmol 1982; 17(5): 227-230.

10 Koehler M, Sholiton D. Spontaneous hyphema resulting from warfarin. Ann Ophthalmol 1983; 15(9): 858-861.

11 Levine MN, Raskob G, Hirsh J. Risk of haemorrhage associated with long term anticoagulant therapy. Drugs 1985; 30(5): 444.

12 Lewis H, Sloan SH, Foos RY. Massive intraocular hemorrhage associated with anticoagulation and age-related macular degeneration. Graefes Arch Clin Exp Ophthalmol 1988; 226(1): 59-64.

13 Kang TS, Lord K, Kunjukunju N. Spontaneous choroidal hemorrhage in a patient on dabigatran etexilate (pradaxa). Retin Cases Brief Rep 2014; 8(3): 175-177.

14 Nguyen TM, Phelan MP, Werdich XQ, Rychwalski PJ, Huff CM. Subconjunctival hemorrhage in a patient on dabigatran (Pradaxa). Am J Emerg Med 2013; 31(2): 455e3-455e.

15 Wang K, Ehlers JP. Bilateral spontaneous hyphema, vitreous hemorrhage, and choroidal detachment with concurrent dabigatran etexilate therapy. Ophthalmic Surg Lasers Imaging Retina 2016; 47(1): 78-80.

16 Jun J, Hwang J. Association of rivaroxaban anticoagulation and spontaneous vitreous hemorrhage. JAMA Ophthalmol 2015; 133(10): 1184-1186.

17 Sakaeda T, Tamon A, Kadoyama K, Okuno Y. Data mining of the public version of the FDA adverse event reporting system. Int J Med Sci 2013; 10(7): 796-803.

18 Zink R Disproportionality analysis and its application to spontaneously reported adverse events in pharmacovigilance. Statistical Discovery. Available at: http://www.jmp.com/content/dam/jmp/documents/en/ white-papers/disproportionality-105686.pdf (accessed on 29 September 2015).

19 Eliquis (apixaban) FDA Approval History-Drugs.com. (n.d.). Available at: http://www.drugs.com/history/ eliquis.html (Retrieved on 11 March 2016).

20 Mason J, Frederick P, Neimkin M, White M, Feist R, Thomley $\mathrm{M}$ et al. Incidence of hemorrhagic complications after intravitreal bevacizumab (avastin) or ranibizumab (lucentis) injections on systemically anticoagulated patients. Retina 2010; 30(9): 1386-1389.

21 Giansanti F, Eandi C, Virgili G. Submacular surgery for choroidal neovascularisation secondary to age-related macular degeneration. Cochrane Database Syst Rev 2009; 15(2): CD006931.

22 Almenoff J, Tonning JM, Gould AL, Szarfman A, Hauben M, Ouellet-Hellstrom R et al. 2005Perspectives on the use of data mining in pharmacovigilance. Drug Saf 28(11): 981-1007. 